\title{
Aspectos generales de la homeopatía
}

\author{
Marcia Avello $L^{a}$, Cristian Avendaño $0^{\text {1b }}$, \\ Sigrid Mennickent $\mathrm{C}^{\mathrm{la}}$.
}

\section{General aspects of homeopathy}

\begin{abstract}
Homeopathic medicine is a type of therapy that appeared in Europe at the end of the eighteenth century. At the present time, it is widely accepted in developed countries as a form of alternative medicine. In Chile, health regulation includes homeopathy as pharmaceutical products and homeopathy is also considered a form of complementary medicine, that is well accepted by the public. The scientific rationale of homeopathy is based on an empiric type of thought that goes from the general to the particular. The symptoms that are valued are those that are particular to each sick individual. It uses diluted solutions of plants, minerals, animals and even venoms. There are basically two hypotheses to explain its mechanisms of action: The "immunological memory" and the "memory of water" or the transmission of electromagnetic information of the water. There still is needed to perform new studies to scientifically assess homeopathy and its usefulness, as an accepted alternative therapy (Rev Méd Chile 2009; 137: 115-20).
\end{abstract}

(Key w ords: Complementary therapies; Homeopathy; Spiritual therapies)

Recibido el 13 de marzo, 2008. Aceptado el 20 de mayo, 2008.

Departamento de Farmacia, Facultad de Farmacia, Universidad de Concepción.

aQuímico Farmacéutico, Magíster en Ciencias Farmacéuticas, Universidad de Concepción

${ }^{\mathrm{b}} \mathrm{Químico}$ Farmacéutico

L a medicina homeopática es un método terapéutico que apareció en Europa a finales del siglo XVIII, anterior a acontecimientos científicos relevantes como la introducción del método experimental en farmacología y fisiología, la teoría microbiana, celular, atómica, de la evolución y el positivismo. Este método se originó a partir de los trabajos y observaciones del médico alemán Samuel C. Hahnemann (1755-1843) ${ }^{1}$.

Hoy, esta medicina tiene una gran aceptación en los países desarrollados, particularmente en Alemania, Francia y Bélgica, la homeopatía es aceptada como medicina complementaria, sin embargo, tiene detractores y defensores que discuten su reconoci-

Correspondencia a: Marcia Avello L Departamento de Farmacia, Facultad de Farmacia, Universidad de Concepción. Casilla 237, Concepción, Chile. Fono: 56-41-2204523. Fax: 56-41-2207086. E mail: maavello@udec.cl miento. Existen estudios que proporcionan evidencia de la efectividad de los remedios homeopáticos. Uno de los estudios a favor de la homeopatía se publicó en 1991 en el British Medical Journal; de 107 ensayos controlados, 81 mostraron que la terapia homeopática era efectiva, 24 determinaron que no tenían efectos, y dos no eran concluyentes ${ }^{2}$.

Según la Organización Mundial de la Salud (OMS, 2002), en una publicación relacionada con la primera estrategia global sobre la medicina tradicional, señala que en muchos países desarrollados, determinadas medicinas complementarias (MCA) son muy populares. Varios informes gubernamentales y no gubernamentales indican que el porcentaje de la población que ha utilizado la MCA es de 46\% en Australia, 49\% en Francia y $70 \%$ en Canadá y encuestas realizadas a 610 médicos suizos demostró que $46 \%$ había utilizado alguna forma de MCA, principalmente la homeo- 
patía y la acupuntura ${ }^{3}$. La OMS está especialmente interesada en apoyar el desarrollo de estas prácticas, fomentando el uso apropiado, seguro y eficaz de la medicina tradicional y complementaria.

En Chile se han desarrollado normativas de regulación de los medicamentos, en que los remedios homeopáticos se incluyen dentro de la categonía de productos farmacéuticos y de regulación de la práctica homeopática como profesión auxiliar de la salud (2004), donde se considera a la homeopatía como una práctica médica alternativa o complementaria de la medicina convencional, que ha sido acogida en forma inveterada por los habitantes del país, siendo su utilización de reconocimiento nacional e internacional y de gran beneficio para quienes recurren a este método terapéutico ${ }^{4}$. Según el químico farmacéutico José Paredes, director técnico del Laboratorio Hochstetter, el uso de esta medicina ha aumentado significativamente en las últimas décadas. En una investigación realizada por un alumno de la carrera de Química y Farmacia de la Universidad de Concepción, en un internado realizado en Oficina de Farmacia en Ancud, se observó que tanto las prescripciones y salida de medicamentos homeopáticos eran considerables ${ }^{5}$.

Concepto. Homeopatía, etimológicamente viene del griego omais =igual y pathos =sentimiento, es un método terapéutico de base científica que persigue la curación de las personas a través de determinadas sustancias de origen natural. Éstas, llamadas "remedios", se prescriben conforme a la ley de la similitud. Consiste en administrar al paciente sustancias en dosis infinitesimales y que, en un sujeto sano, en dosis ponderables producirán los mismos síntomas que en enfermedad tratada (efecto paradojal). Se basa en tres principios básicos:

1. Ley de semejanza o similitud (origen hipocrático). El Dr. Angel Marzetti, en su libro "La Homeopatía, Medicina del Porvenir", habla sobre el reconocimiento involuntario de la existencia de este principio, ejemplificando; "Diez o veinte gramos de sulfato de sodio producen una diarrea acuosa con poco dolor. Diez o veinte centígramos o aun una cantidad mucho menor, curan una diarrea semejante" ${ }^{\prime 6}$.

2. Individualización del enfermo y no de la enfermedad. Basado en las patogenesias, que es el conjunto de perturbaciones que la sustancia en dosis ponderables genera en el experimentador.
3. Dosis infinitesimales o microdosis de sustancia activa. Por medio de diluciones y dinamizaciones se obtiene el remedio homeopático ${ }^{6}$.

Su método científico, basado en el pensamiento empirista, va de lo general a lo particular, es decir, los síntomas valorables en la enfermedad son los "propios" de cada enfermo, en particular. Existe una aceptación plena de la noción de la physys (naturaleza del ser) con su esfuerzo curativo, considerándola espontánea, autodeterminante y con múltiple capacidad defensiva ${ }^{6}$.

Cabe señalar que el tratamiento con homeopatía no conlleva efectos adversos, ni contraindicaciones, lo que constituye una ventaja en relación a otras medicinas.

Historia. El tratamiento de los enfermos con remedios que producen cuadros reaccionales similares a los que aparecen en las enfermedades naturales, fue conocido hace más de mil años antes de nuestra era por los indios y los chinos?

Hipócrates, de la isla griega de Cos (460 a 377 a.C.), estructura las bases de la Ley de la Semejanza; y escribió: "La enfermedad es producida por los semejantes, y por los semejantes que la producen, el paciente regresa de la enfermedad a la salud. Así, aquello que produce la estranguria que no existía, cura la estranguria que existe. La tos como la estranguria es causada y eliminada por las mismas cosas"8.

Una figura fundamental en el pensamiento empirista fue Paracelso (1493-1541) que arremete contra la práctica médica de su época y plantea la necesidad de retomar el concepto de physys presocrático, al que él llama "naturaleza", y resalta su poder curativo. Establece la causa de las enfermedades en relación a los astros, a los cambios meteorológicos y a la propia esencia del hombre, así como cree que la causa de las enfermedades se encuentra en la naturaleza. Planteó que la ley de semejanza utilizando sustancias en soluciones diluidas era capaz de curar enfermedades, por lo que empleó la karena, que es igual a la veinticuatroava parte de una gota ${ }^{8}$.

Pero la homeopatía propiamente tal, surge en Alemania con Samuel Hahnemann (1755-1843), creador de la homeopatía a finales del siglo XVIII. Se graduó de médico en la Universidad de Erlangen en 1779. Además del ejercicio profesional, era un estudioso de las ideas filosóficas, con gran admira- 
ción por Kant; profundo conocedor de idiomas, era traductor de obras científicas al alemán. Al traducir del inglés la Materia Médica de Cullen, observa las hipótesis contradictorias sobre la acción de la quinina, luego constata que la administración reiterada de quinina coincide en la sintomatología con la de aquellos enfermos que cura. La experimenta en él mismo y en sus más allegados, dando dosis diarias por varios días, y encontró que producía una fiebre similar a la que ocurre en la malaria que curaba cuando se empleaba como tratamiento ${ }^{8}$.

Al seguir los pasos del método científico de modo irreprochable: observación, hipótesis y experimentación, además de la comprobación de las "patogenesias", se deduce que el método de Hahnemann está basado en la experiencia, que no es ciega o casual, sino que adquiere el carácter de experimento científico, en el que descubre y comprueba la ley de semejanza ${ }^{5}$.

Publicó tres obras sobre homeopatía; El Organón de la Medicina, Las Enfermedades Crónicas, y La Materia Médica Pura. Con Hahnemann nace definitivamente la Medicina Homeopática, que es el estudio, diagnóstico y tratamiento del terreno humano enfermo, representado por la estructura del organismo con sus procesos de orden psíquico que le son propios, sus permanentes cambios fisiológicos y las alteraciones patológicas que tuvieren lugar, y conforme a la ley de la semejanza ${ }^{8}$.

Hahnemann postulaba que el conjunto de síntomas y signos de una enfermedad constituía el esfuerzo concentrado de todo el organismo para curarse a sí mismo y que el remedio sólo inicia 0 refuerza de alguna manera el intento de sanar ${ }^{9}$.

Fuerza vital. El Dr. Samuel Hahnemann toma el concepto de physys y le da el nombre de "fuerza vital", básicamente es el poder de los seres vivos a sanarse por sí mismos, la que al ser estimulada, tiende a producir una modificación en el terreno, ya sea con una sintomatología especialmente particular o se producirá la corrección de una alteración que ha estado en ese terreno, con la consiguiente desaparición de los síntomas que estaban presentes. Por lo cual con fines de tratamiento, la estimulación depende de las características y selectividad de la energía, o sea, el remedio. Desde la perspectiva de Hahnemann, la etiología de las enfermedades carece de importancia; "De poco servirá conocer las causas de la enfermedad, ya que su origen reside en un desequilibrio de la energía vital del enfermo y la curación sólo puede obtenerse restableciendo ese equilibrio" ${ }^{8}$. Para el creador de la homeopatía; "La naturaleza es ciega y obra sin inteligencia. Por tanto, no hay que imitarla sino ayudarla utilizando la inteligencia y la razón" 6 .

Diagnóstico y tratamiento. Para el diagnóstico homeopático se utiliza el examen minucioso y directo del paciente mediante anamnesis y examen físico. A partir de aquí, el médico busca la sustancia medicinal cuyos signos y síntomas, obtenidos en experimentación, sean lo más parecido posible a los de la enfermedad del paciente. Un ejemplo común en atención primaria es la fiebre de los niños en edad preescolar. Las manifestaciones típicas son mejillas enrojecidas, boca seca, pupilas dilatadas, agitación e incluso alucinaciones; un cuadro de síntomas muy similar puede ser inducido en un niño sano administrando belladona (síntomas atropínicos). El tratamiento homeopático que recibiría el niño enfermo sería un remedio a partir de belladona en dosis infinitesimales (dosis mínima efectiva) ${ }^{10}$.

Por otro lado, tal como se desarrolló tradicionalmente, la homeopatía es un arte holístico que observa el cuadro sintomático de una persona en forma integral, incluyendo información psicológica, emocional, física y hereditaria. Por lo que se usan los "remedios constitucionales" que van de acuerdo al biotipo de la persona, esto en concordancia con que la homeopatía "trata enfermos no enfermedades". Cuando se practica de esta forma, a menudo es llamada "homeopatía constitucional", debido a que intenta dirigir la constitución (salud implícita) de una persona, en lugar de los síntomas superficiales. Ambos sistemas se han estudiado científicamente, aunque la homeopatía orientada a la enfermedad ha recibido más atención por la simple razón de que es más fácil de estudiar².

Existen remedios constituidos por una sola sustancia o remedio simple, y remedios compuestos que contienen distintas sustancias, las cuales tienen indicación terapéutica más específica a diferencia de los simples. Los remedios con un solo constituyente tienen una aplicación en diversas patologías a la vez, que innecesariamente están relacionadas con su ubicación en determinados sistemas del cuerpo, ni tampoco una relación estrictamente fisiopatológica de la enfermedad, como por ejemplo, el remedio 
"berberis" se podría utilizar al mismo tiempo para afecciones a los riñones como para determinadas enfermedades articulares. Sin embargo, la homeopatía se prescribe de acuerdo a la sintomatología del paciente, es decir, son medicamentos que se prescriben basados en un repertorio clínico homeopático que incluye, además, los síntomas específicos mentales y locales que experimenta el paciente, por ejemplo, "disnea, con deseo de abrir ventanas", "melancolía", "sensación de nudo en hipocondrio izquierdo", etc., sintomatología que es obtenida desde la experimentación con sustancias potencialmente terapéuticas en personas sanas. En cambio, los medicamentos homeopáticos compuestos, tienen una indicación para patologías determinadas como el "homoio 47" para urticaria y afecciones a la piel, o "mediform 89" indicado para colon imitable y otras afecciones del tracto gastrointestinal.

Disoluciones homeopáticas. Como se mencionó anteriormente, la segunda ley de la homeopatía requiere que el tratamiento homeopático sea diluido para el máximo efecto. Hahnemann desarrolló técnicas para controlar la concentración, o disolución, de las sustancias para crear los remedios homeopáticos. Primero, tomó la sustancia y la conservó en un solvente, normalmente alcohol. Las sustancias que usó fueron plantas y minerales. Después de dejar que la sustancia reposara por un mes, vertió el líquido, lo que se convirtió en la «disolución madre.» Luego, tomó una gota de la disolución y le añadió 99 gotas de alcohol puro. Mezcló el líquido precipitando el contenedor sobre una superficie dura, un proceso llamado «sucusión». Los médicos homeopáticos creen que la dinamización es esencial para crear un remedio efectivo.

El primer paso crea un remedio con una disolución de una parte en $10^{2}$ o 100 . Esta disolución será anotada por «c,» de centesimal (indicando una disolución de dos factores de 10), o por los términos 2x o D2 (x y D, cada uno, indican un factor de 10). La disolución continúa, siempre añadiendo una parte de la tintura a 99 partes de alcohol y haciendo dinamización a cada paso. Dicho proceso realizado seis veces conlleva a un remedio 6c (o 12x o D12) y así sucesivamente.

Algunas veces se fabrican los remedios homeopáticos con sustancias que son insolubles. En este caso, se muelen, se mezclan con lactosa y luego se convierten en remedios.
El proceso completo de creación de los remedios homeopáticos se llama spotenciación», basados en la teoría de que cada disolución sucesiva hace al remedio más potente ${ }^{2}$.

Hoy, se pueden encontrar remedios homeopáticos que consisten de pequeñas pastillas blancas de azúcar de leche que han absorbido la solución potenciada, llamadas glóbulos y microglóbulos (en dilución C-200). Otros remedios se encuentran en forma de solución para gotas e inyectables, comprimidos y cremas para usar externamente.

La farmacopea homeopática reconoce hoy más de 2.000 remedios y cada día se descubren nuevas plantas (hojas, raíces y frutos), componentes que provienen del reino mineral (tierra y minerales) y elementos que provienen del reino animal, entre otros algunos venenos ${ }^{9}$.

Formas especiales de remedios homeopáticos. Además de los remedios homeopáticos estándares que usan sustancias en relación a la patogenesia (síntomas y signos provocados por la sustancia), hay otras dos formas especiales de remedios homeopáticos que usan sustancias específicamente relacionadas con la enfermedad. Los remedios isopáticos por un lado, que se hacen de la sustancia real que causa la enfermedad. Por ejemplo, la pelusa de gato, homeopáticamente preparada (conteniendo cero moléculas de pelusa de gato), podńa usarse para tratar la alergia a los gatos. Y los remedios nosodes, que se hacen de tejidos de animales infectados o secreciones corporales. Las glándulas infectadas de tuberculosis de una vaca podnían diluirse homeopáticamente para crear un remedio para la tuberculosis humana ${ }^{2}$.

Posibles mecanismos de acción. La homeopatía no se trata de una terapéutica más, sino de toda una nueva concepción acenca de la salud y la enfermedad. Con esta terapéutica, Hahnemann se adelantó a su tiempo, al presentar una metodología avanzada para la ciencia de su época y aun de la nuestra, pero hoy es posible realizar con ella investigaciones en alguno de sus aspectos, mediante equipos interdisciplinares de campos como la biología molecular, la inmunología o la físico-química ${ }^{11}$.

Existen básicamente dos hipótesis para explicar el mecanismo por el que actúa la homeopatía, una de ellas es la hipótesis de "asistencia inmunológica" en la terapia antihomotóxica, que goza de demostración. Al administrarse el medicamento homeopático, en pri- 
mer lugar se enfrentan directamente y de forma inespecífica a los monocitos/macrófagos. Tras la fagocitosis, los macrófagos devuelven un motivo (o segmento) de aminoácidos (una cadena de 5 a 15 aás.) de las sustancias a su superficie. Aquí ligan al complejo MHC (complejo mayor de histocompatibilidad). De este modo los motivos se hacen reconocibles para los linfocitos ("inmaduros"-Th0-) aún indiferenciados. Éstos toman los motivos de aminoácidos, convirtiéndose así en células Th3 reguladoras. Luego, viajan por los vasos linfáticos a los nódulos linfáticos más cercanos y allí forman clones celulares "con motivos", que entran en el torrente sanguíneo a través de las vénulas poscapilares y se reparten por todo el organismo a través de la circulación. En las áreas disreguladoras, especialmente en zonas de inflamación, atraen a las células Th3 gracias a un mecanismo químico (factores del complemento, quimiocinas, etc.) en función de sus motivos, pueden reconocer a los linfocitos inflamatorios (células T4 y sus subpoblaciones: Th1 y Th2). Para ello es suficiente que las secuencias sean similares (principio de similitud de la medicina antihomotóxica), a fin de que las células Th3 se estimulen para secretar la citosina TGF- $ß$ (factor transformante del crecimiento tisular beta) y en menor medida la IL-4 e IL-10. El TGF-ß es la citosina antiinflamatoria más potente del organismo. Esta citosina inhibe a las células T4 y sus células auxiliares. Al mismo tiempo, las células Th2 refuerzan su propia desactivación liberando IL-4 e IL-10, de esta forma, refuerzan considerablemente la función antiinflamatoria del TGF-ß. Simultáneamente, los linfocitos B son estimulados para realizar la síntesis de inmunoglobulinas. Hahnemann, proponía diluir las sustancias tóxicas hasta el punto donde sólo tuvieran un efecto de "estimulación". Empíricamente, estaba descubriendo un sistema terapéutico basado en la modulación inmunológica ${ }^{12}$.

Queda mencionar que la reacción de asistencia inmunológica solamente se puede desarrollar en el rango de dosis bajas de antígeno desde diluciones homeopáticas D1 hasta aproximadamente la D14, por lo que para las demás diluciones, no se podría explicar su efecto terapéutico, con esta teoría.

Otra hipótesis existente de la acción del medicamento homeopático, es el de la "memoria del agua" o de la transmisión de una información de naturaleza electromagnética en el agua. De alguna manera, las cualidades del agua son modificadas en los procesos de preparación del medicamento, sobre todo por efecto de la dinamización, de tal forma que queda impregnada de la información que le transmite la tintura madre en las sucesivas diluciones y sucusiones, constituyendo los elementos que actúan farmacológicamente en el organismo ${ }^{12}$. Esto podría explicar la persistencia de la actividad biológica de las diferentes sustancias químicas más allá del límite físico representado por el Número de Avogadro ("fenómeno homeopático").

El Dr. Gabriel Hemán Gebauer explica que el mecanismo de acción se encuentra en la información que lleva el remedio homeopático y que es la clave aquello que permite que desde la memoria del soluto»(tintura madre) se pase a constituir la memonia del agua". Las informaciones no pueden desaparecer porque son energías no degradables, es decir, formas de energía que no participan del estado de equilibrio -que es el estado de degradación de la energía-. Por eso hemos hablado de la información como de una energía que es, la mayor parte del tiempo, una energía potencial, salvo en el momento mismo de su expresión, o sea, cuando ese sistema aislado se abre y se produce intercambio con el medio. Agrega, "La enfermedad es desorden, la curación no podrá ser sino la recuperación del orden perdido. El tratamiento que pretenda ser curativo deberá aportar orden, exactamente el orden que el organismo enfermo que sea el caso necesita". "La información es orden porque la información es, por un lado, cualitativamente específica y es, por otro lado, energía capaz de ordenar activamente el sistema que sea capaz de recogerla"13.

Las teonías de la homeopatía parecen contradecir las leyes básicas de la física y química, en relación a las diluciones más altas (desde D30 a C200), parece razonable insistir en que la homeopatía pasa un estándar más alto de evidencia que otras formas de la medicina alternativa. Pero hay que dejar en claro, la homeopatía nació en un entomo precientífico hace dos siglos, pero se trata de una medicina nacida de la experimentación y no de la abstracción teórica ${ }^{15}$. Se considera que la totalidad del organismo es la causa de todos los cambios que en él se producen. En este sentido, la homeopatía es el modelo de una doctrina médica integradora ${ }^{6}$.

Asociaciones con otras terapias. Es común el uso conjunto de la homeopatía con otras medicinas como la fitoterapia (productos fitoterápicos pre- 
elaborados, elaborados y hierbas medicinales en su estado natural), con el fin de aumentar la efectividad del tratamiento en los pacientes. También es frecuente la asociación con medicamentos alopáticos. En relación a este punto sólo el profesional médico está autorizado para la prescripción, por lo tanto, es de esperar que esta asociación no sea frecuente, pero la utilización concomitante de medicamentos alopáticos con remedios de homeopatía, es totalmente viable. La homeopatía por un lado, estimula la capacidad curativa interna a través del reconocimiento y estimulación de ciertas reacciones que forman parte del sistema inmunitario o de defensa. Por otro lado, la alopatía actúa en la sintomatología del paciente y en contrarrestar el mecanismo patológico que provoca la enfermedad. En consecuencia, no existe inconveniente alguno para la asociación de estos sistemas terapéuticos, ni mucho menos con fitoterapia, dado que actúan en diferentes niveles del organismo. No obstante sí generará un inconveniente para el médico, ya que no podrá evaluar la respuesta del paciente al tratamiento con homeopatía.

La importancia y desarrollo que ha alcanzado la homeopatía en Chile es considerable en los últimos años. Por lo tanto, es indispensable conocer las características de esta práctica en nuestro país.

\section{REFERENCIAS}

1. De SAgREDa JE. Homeopatía, Interpretación Histórica de un Debate Interminable. Facultad de Farmacia, Universidad de Barcelona, OFFARM. 2006; 25: 86.

2. http:/healthlibrary.epnet.com/

3. Publicación Oficial de la Organización Mundial de la Salud (OMS). Estrategia de la OMS sobre la Medicina Tradicional 1002-2005. Ginebra, 2002; 11.

4. Subsecretaria de Salud Pública. Departamento de Asesoría Jurídica. Decreto del Reglamento para el Reconocimiento y Regulación a la Práctica Homeopática como Profesión Auxiliar de la Salud. Santiago, Chile, 2004; 1.

5. HochstetTeR F. Homeopatía y otras Terapéuticas Complementarias. $2^{\mathrm{a}}$ ed. Santiago, Chile, 1972; 11.

6. Baulester Sanz A, Sanz Franco MJ, Galan Grau E. Formación Médica Continuada en Atención Primaria. Homeopatía, Fundamentos Científicos. Centro de Salud de Nazaret y de Cullera. Valencia, España, 1999; 72.
En Chile, la homeopatía es practicada tanto por médicos como por "no-médicos" que practican la medicina naturista. Estos especialistas sin un título profesional son denominados por la legislación chilena como auxiliares de la salud. Además, no existe un título reconocido de homeópata, por lo que la mayonía de los practicantes son autodidactas en su formación homeopática, incluyendo los profesionales médicos. Existen cursos de acercamiento que entregan los laboratorios del rubro de homeopatía, no obstante, no hay regulación que controle su enseñanza. A pesar de ello, desde 1941 existe una legislación específica reguladora de la homeopatía dentro del esquema sanitario general y sus medicamentos se rigen dentro de la ley como productos farmacéuticos. Se ha regulado bajo qué condiciones se puede ejercer la práctica homeopática, relacionado con los conocimientos mínimos que debe tener el homeópata, las actividades que puede llevar a cabo y requisitos de infraestructura del establecimiento de la consulta.

Con el fin de que este sistema pueda ser considerado ampliamente como una terapia alternativa o complementaria y de mejorar su utilización por parte del área de la salud, es necesario profundizar en el estudio de la homeopatía y su práctica, sobre todo los profesionales de la salud, ya que son éstos los capacitados para tratar enfermedades.

7. NeiJing. Cuestiones Básicas de Medicina Interna (en: http//:es.wikipedia.org).

8. Barros-St. Pasteur J. Homeopatía: Medicina del Terreno. Ediciones de la Biblioteca. Universidad Central de Venezuela, Caracas, 1977; 17-22.

9. Baldester Sanz A, Gosalbez E, Baleester Fernández R. Experiencia de una Consulta de Homeopatía Pediátrica en un Centro de Salud. Centro de Salud de Nazaret y de Cullera. Valencia, España, 2003; 55.

10. Cuesta R. El Medicamento Homeopático y su Normativa Legal. Farmacéuticos del CGCOF. España, 1998; 55.

11. Catálogo de Productos Heel Chile. Terapia de Rutina. Productos farmacéuticos Heel Chile Ltda. Santiago de Chile, 2002; 6 -7.

12. Gebauer GH. Una Nueva Teoría acerca de las Diluciones Homeopáticas. Santiago. Chile, 2002; 7.

13. Calleja Peredo C. Especialista Universitario en Homeopatía por la Universidad de Valladolid, 2002; 9. 